\title{
Psychological Effects of Lockdown Measures for the COVID-19 Outbreak in Patients with Systemic Lupus Erythematosus
}

\section{Luca Quartuccio, (D) ${ }^{1,2, *}$ Ginevra De Marchi, ${ }^{1} *$ Danila Azzolina, ${ }^{3}$ Elisabetta Maresio, ${ }^{4}$ Donatella Colatutto, ${ }^{1,2}$ Marco Binutti, ${ }^{1,2}$ Marinella Monte, ${ }^{5}$ Silvia Gallipoli, $\mathbb{D}^{6}$ Federica Zobec, ${ }^{6}$ Corrado Lanera, ${ }^{3}$ Giulia Lorenzoni, ${ }^{3}$ Alvisa Palese, ${ }^{2,7}$ Marco Silano, ${ }^{8}$ Salvatore De Vita, ${ }^{1,2}$ Dario Gregori $\mathbb{D}^{3}$}

'Rheumatology Clinic, Department of Specialist Medicine, ASUFC, Udine, Italy; ${ }^{2}$ Department of Medicine (DAME), University of Udine, Udine, Italy; ${ }^{3}$ Unit of Biostatistics, Epidemiology and Public Health, Department of Cardiology, Thoracic and Vascular Sciences, University of Padova, Padova, Italy; ${ }^{4}$ Prochild Onlus, Unit of Psychotherapy and Psychology, Trieste, Italy; ${ }^{5}$ Associazione Malati Reumatici (A.Ma.Re.), Udine, Italy; ${ }^{6}$ Zeta Research s.r.l., Trieste, Italy; ${ }^{7}$ School of Nursing, Department of Medicine, University of Udine, Udine, Italy; ${ }^{8}$ Unit of Human Nutrition and Health, Department of Food Safety Nutrition and Veterinary Public Health, Istituto Superiore di Sanità, Rome, 0016I, Italy

*These authors contributed equally to this work

Correspondence: Luca Quartuccio Department of Medicine (DAME), University of Udine, Udine, Italy

Tel +390432559352

Fax +39 0432559472

Email luca.quartuccio@uniud.it
Objective: To compare the psychological impact of the lockdown measures contrasting the COVID-19 outbreak between systemic lupus erythematosus (SLE) and general population.

Patients and Methods: From July 15th to August 15th 2020, a retrospective survey referring to the period March 9th to May 18th 2020 was administered to SLE patients and the results of the survey, called LEPRE (Lupus Erythematosus PREsto) study, were compared with those from the PRESTO (imPact of quaRantine mEasures againST cOvid19) project, the same survey provided to the general population. Consecutive patients $>18$ years old affected by SLE and regularly followed in a single rheumatologic centre were involved. Primary outcome was to compare the scores of the Impact of Events Scale-Revised (IES-R), the General Health Questionnaire 12 (GHQ-12) and the Center for Epidemiological Depression Scale (CES-D) between patients and general population.

Results: A total of 64 patients completed the survey. After a propensity score matching, they were compared to 128 people from PRESTO project. The median age among patients was 43 years (I-III interquartile range 35-54.5), 88\% were female and 100\% Caucasian. IES-R [(score $>23: 57 \%$ (34) vs 49\% (58)], GHQ-12 [(score>13: 85\% (52) vs 88\% (106)], and CESD [(score $>15: 45 \%$ (28) vs 40\% (46)] scores were not statistically different between patients and controls $(\mathrm{p}>0.05)$.

Conclusion: Restrictive measures for COVID-19 pandemic had no greater impact on patients with SLE than in the general population. Strategy for coping to the SLE might be useful during lockdown measures and may be helpful for other chronic conditions.

Keywords: systemic lupus erythematosus, COVID-19, psychological distress

\section{Introduction}

COVID-19 is a systemic viral disease currently spreading as a pandemic. ${ }^{1}$ A more severe course and prognosis of COVID-19 in some autoimmune diseases, such as systemic lupus erythematosus (SLE) and vasculitis, and for some immunosuppressive agents or higher doses of glucocorticoids has been reported. ${ }^{2}$

SLE is the model of systemic autoimmune disease, that is characterizes by the occurrence in young people, chronic course, increased susceptibility to infections, damage accrual due to the disease itself and long-term glucocorticoid therapy and immunosuppressive treatments, and comorbidities. Furthermore, SLE is a chronic disease that deeply affects multiple dimensions of young patients' lives, with fatigue and chronic pain being the most frequent symptoms, with a profound effect on quality of life, despite treatments. ${ }^{3,4}$ On the other hand, chronic diseases 
affecting the humoral immune system show a great impact on mental health. In fact, a stronger association with psychiatric disorders and suicidal behavior has been recently highlighted for joint exposure to primary humoral immunodeficiencies and autoimmune disease. ${ }^{5}$ Thus, a pandemic is a trigger that can influence the mental health of patients living with SLE.

The PRESTO (imPact of quaRantine mEasures againST cOvid19) project is a survey that investigated the impact of the Italian government measures adopted between March 9th and May 18th 2020. This project was sponsored by SANV Unite (Feeding, Nutrition and Health) of the Italian Istituto Superiore di Sanità, the Unit of biostatistics, epidemiology and public health of Department of cardio-thoracic-vascular Science and public health (DCTV) of Padua University and by Psychotherapy and Psychology Unit of Prochild Onlus, with technical support by Zeta Research S.r.l. The project was based on a broad review of studies which reported negative psychological effects of quarantine, including post-traumatic stress symptoms, confusion, and anger. Stressors included longer quarantine duration, infection fears, frustration, boredom, inadequate supplies, inadequate information, financial loss, and stigma. ${ }^{6}$

Our investigation, namely the Lupus Erythematosus PRESTO (LEPRE) study, aimed to evaluate the psychological impact of lockdown measures against COVID-19 on patients with SLE as compared to the general population participating in the PRESTO project.

\section{Patients and Methods}

From July 15 th to August 15th 2020, consecutive patients $\geq 18$ years old affected by SLE classified according to the 2019 ACR criteria $^{7}$ and regularly followed at our Rheumatology Clinic, Academic Hospital of Udine, were asked to anonymously complete the online questionnaire referring to the quarantine period March 9th to May 18th 2020. All patients who were involved had been taking stable treatment for at least 6 months and they were in remission or in low disease activity state. ${ }^{8}$ Patients with pre-existing neuropsychiatric disorders were excluded. During the lockdown measures, the patients had full access to the hospital for urgent visits, and could communicate with medical staff via telephone or email. The survey collected socio-demographic data and focused on COVID-19 concerns, emotional impact, self-perception, and change in habits during quarantine for the COVID19 outbreak. The psychological impact was measured using the Impact of Events Scale-Revised (IES-R), ${ }^{9}$ the General Health Questionnaire 12 (GHQ-12), ${ }^{10}$ and the Center for Epidemiological Studies Depression Scale (CES-D). ${ }^{11}$

In detail, the IES-R was designed as a measure of posttraumatic stress disorder (PTSD) symptoms, and is a short, easily administered self-report questionnaire. It is best used for recent and specific traumatic events. It has 22 questions, 5 of which were added to the original Horowitz (IES) to better capture the DSM-IV criteria for PTSD. The IES-R score was categorized into 4 classes according to a recent publication in the field: 0-23 (normal), 24-32 (mild psychological impact), 33-36 (moderate psychological impact), and $\geq 37$ (severe impact). ${ }^{12}$ To assess psychological distress, the GHQ-12 was employed, using a cut-off at 14 points to identify psychological distress. ${ }^{13}$ Finally, depression was screened using the CES-D. Three classes of symptoms severity were considered: 0-15 (no/mild depressive symptoms), 16-23 (moderate depressive symptoms), and 24-60 (severe depressive symptoms). ${ }^{11}$

Results from the LEPRE project were compared with those reported by the general population (PRESTO project), with further sub analyses by including or not people declaring chronic diseases. The PRESTO project is a survey started on the 20th of March 2020 with the aim of describing lifestyle habits and the prevalence of psychological discomfort among the Italian population during the COVID-19 lockdown. It was web-based, using Lime Survey, and it was disseminated via messaging apps (eg, WhatsApp) and social networks (eg, Facebook).

Stakeholders other than physicians were involved in the study: health professionals (AP), psychologists (EM), nutritionists (MS), epidemiologists and biostatisticians (DG, DA, CL, GL), and patients (MM) were involved in planning the study and interpretation of the results.

Data were summarized according to LEPRE versus PRESTO groups (for the best comparison only people coming from Friuli Venezia Giulia region of Italy were selected) and reported as a percentage and absolute number. The Pearson Chi-Square test was performed to compare the groups together with the Fisher exact test wherever appropriate.

A propensity score was estimated to match the LEPRE cases to the PRESTO responders with a ratio of 2 versus $1 .^{14}$

To perform clustering analysis on categorical data, the Multiple Correspondence Analysis (MCA) results can be used to transform categorical variables into a set of few 
continuous variables (the latent dimension). The cluster analysis can then be applied to the results. In this case, the MCA can be considered as pre-processing steps that allow computing clustering on categorical data.

A Factorial Agglomerative hierarchical clustering analysis has been then carried out on the MCA results. The distance between MCA loadings has been computed via Manhattan measure using the Ward method to identify the hierarchical partition. The data partition has been represented in a cluster dendrogram. The cluster membership together with the individual loading has been represented in a factor map with the $95 \%$ confidence ellipses around centroids.

The features discriminating the clusters according to the Chi-square significance $($ alpha $<0.05)$ have been selected and summarized (Figures S1 and $\underline{\text { S2) }}$.

A significance level of 0.05 has been considered for the analyses. Computations have been performed with the $\mathrm{R}$ 3.6.2 system and the Factominer, Matchit, and Factoextra packages.

\section{Results}

Among 110 patients who were asked to participate in the study, 64 patients anonymously completed the survey and were included in the LEPRE study. They were compared to 1114 unselected people living in the same geographical area of Italy, namely the Friuli Venezia Giulia region. The survey responders' characteristics were different between the LEPRE and PRESTO; the LEPRE group showed a higher percentage of females [88\% (56) vs 61\% (673), $\mathrm{p}<0.001]$ and subjects with lower educational level (prevalently high school) [University: 28\% (18) vs 49\% (548), $\mathrm{p}=0.001]$. The SLE subjects live mainly in detached houses [59\% (38) vs 31\% (348), p<0.001], having access to a private garden [81\% (52) vs 56\% (625), $\mathrm{p}<0.001]$ and also owning a pet [67\% (43) vs $46 \%$ (508), $\mathrm{p}=0.001]$, in comparison with the PRESTO sample living mainly in flats [39\% (25) vs 66\% (740), $\mathrm{p}<0.001]$ (Table 1).

Moreover, a greater percentage of the LEPRE study group had a swab test during the pandemic [19\% (12) vs $4 \%$ (22), $\mathrm{p}<0.001]$. The LEPRE sample practiced less frequent physical activity during the quarantine in comparison with the PRESTO group [27\% (16) vs 43\% (392), $\mathrm{p}=0.016]$ (Table 1).

However, after matching the sample using the propensity score all the standardized mean differences lie in the +-0.1 threshold indicating a suitable balance across groups (Figure S1).
One hundred and twenty-eight matched people from the PRESTO study were compared to the 64 SLE patients. The median age among patients was 43 years (I-III interquartile range 35-54.5), $88 \%$ were female and $100 \%$ Caucasian.

No significant differences were identified in the groups' characteristics after the matching procedure (Table 2).

The psychological impact measured by IES-R [(score $>23: 57 \%$ (34) vs 49\% (58)], GHQ-12 [(score $>13$ : $85 \%$ (52) vs $88 \%$ (106)], and CES-D [(score $>15: 45 \%$ (28) vs $40 \%$ (46)] scores were not statistically different between patients and the general population $(\mathrm{p}>0.05)$ (Table 2).

As regards missing activities, patients with SLE missed playing sports/exercise less than general population $[19 \%$ (12) vs 36\% (46), $\mathrm{p}=0.017)$ ], while they felt more the lack of the company of family and relatives [71\% (45) vs 33\% (42), p<0.001] (Table 3).

Concerning the responders' feelings during the quarantine, COVID-19 concerns, anxiety, and depression rates as well as feelings were not different between patients and general population (Table 4). However, patients perceived more difficulty to find some more

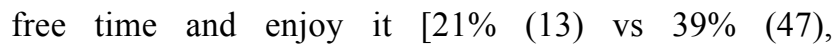
$\mathrm{p}=0.01)]$ and to be able to solve own problems more than usual [(0 vs $11 \%(13), \mathrm{p}=0.02)]$ (Table 4$)$. On the contrary, patients felt less sad or depressed than usual [28\% (17) vs $11 \%(13), \mathrm{p}=0.003]$ in comparison with the PRESTO group (Table 4). Moreover, the PRESTO group less frequently avoided discussions that could remind them of the pandemic $[(37 \%$ (22) vs $56 \%(66)$, $\mathrm{p}=0.02)$ ] (Table 4).

Regarding emotional status, the patients also very rarely or never had the impression that people did not like them [(93\% (56) vs 75\% (87). $\mathrm{p}=0.003)]$ (Table 5).

Finally, no differences were evidenced for dietary habits during the quarantine among groups, except for a greater consumption of eggs per week for the PRESTO group [(median (I-III quartile range): 1 (1-2) vs 2 (1-2), $\mathrm{p}=0.005)]$ (Table S1).

Notably, two sub analyses performed by comparing LEPRE subjects only with people with chronic diseases (Tables S2-S4), or only with healthy volunteers retrieved the same results (Tables S5-S7).

The clusters' characteristics are reported in Table S8: cluster 1 is composed in prevalence of males reporting a 
Table I Descriptive Statistics of the Survey Responders

\begin{tabular}{|c|c|c|c|c|c|c|}
\hline Answer & $\begin{array}{c}\text { N. of } \\
\text { Responders }\end{array}$ & Response & $\begin{array}{l}\text { LEPRE } \\
(\mathbf{N}=64)\end{array}$ & $\begin{array}{l}\text { PRESTO } \\
(N=|| \mid 4)\end{array}$ & $\begin{array}{l}\text { Combined } \\
(\mathrm{N}=1 \mid 78)\end{array}$ & $\begin{array}{c}P \\
\text { value }\end{array}$ \\
\hline \multirow[t]{2}{*}{ How old are you? } & 1178 & $\begin{array}{l}\text { Age, median (I-III } \\
\text { interquartile range) }\end{array}$ & $43.0(35-54.5)$ & $47.0(34-57)$ & $47.0(35-56.8)$ & 0.6 \\
\hline & 1178 & $\begin{array}{c}\leq 29 \text { years } \\
29-65 \text { years } \\
>65 \text { years }\end{array}$ & $\begin{array}{c}16 \%(10) \\
81 \%(52) \\
3 \%(2)\end{array}$ & $\begin{array}{l}19 \%(207) \\
71 \%(791) \\
10 \%(116)\end{array}$ & $\begin{array}{l}18 \%(217) \\
72 \%(843) \\
10 \%(118)\end{array}$ & 0.112 \\
\hline What is your gender & 1176 & $\begin{array}{l}\text { Female } \\
\text { Male }\end{array}$ & $\begin{array}{c}88 \%(56) \\
12 \%(8)\end{array}$ & $\begin{array}{l}61 \%(673) \\
39 \%(439)\end{array}$ & $\begin{array}{l}62 \%(729) \\
38 \%(447)\end{array}$ & $<0.001$ \\
\hline Please specify your nationality & 1178 & $\begin{array}{l}\text { Other } \\
\text { Italian }\end{array}$ & $\begin{array}{c}3 \%(2) \\
97 \%(62)\end{array}$ & $\begin{array}{c}2 \%(20) \\
98 \%(1094)\end{array}$ & $\begin{array}{c}2 \%(22) \\
98 \%(1156)\end{array}$ & 0.445 \\
\hline What is your level of education? & 1178 & $\begin{array}{c}\text { High School } \\
\text { University } \\
\text { Secondary school }\end{array}$ & $\begin{array}{c}70 \%(45) \\
28 \%(18) \\
2 \%(1)\end{array}$ & $\begin{array}{c}51 \%(563) \\
49 \%(548) \\
0 \%(3)\end{array}$ & $\begin{array}{c}52 \%(608) \\
48 \%(566) \\
0 \%(4)\end{array}$ & 0.001 \\
\hline What is your employment status? & 1178 & $\begin{array}{l}\text { Employed } \\
\text { Student }\end{array}$ & $\begin{array}{c}97 \%(62) \\
3 \%(2)\end{array}$ & $\begin{array}{l}92 \%(1029) \\
8 \%(85)\end{array}$ & $\begin{array}{l}93 \%(1091) \\
7 \%(87)\end{array}$ & 0.18 \\
\hline What is your house like? & 1178 & $\begin{array}{c}\text { Flat } \\
\text { Studio flat } \\
\text { Detached house }\end{array}$ & $\begin{array}{c}39 \%(25) \\
2 \%(1) \\
59 \%(38)\end{array}$ & $\begin{array}{c}66 \%(740) \\
2 \%(26) \\
31 \%(348)\end{array}$ & $\begin{array}{c}65 \%(765) \\
2 \%(27) \\
33 \%(386)\end{array}$ & $<0.001$ \\
\hline Can you access a private garden? & 1176 & $\begin{array}{l}\text { No } \\
\text { Yes }\end{array}$ & $\begin{array}{l}19 \%(12) \\
81 \%(52)\end{array}$ & $\begin{array}{l}44 \%(487) \\
56 \%(625)\end{array}$ & $\begin{array}{l}42 \%(499) \\
58 \%(677)\end{array}$ & $<0.001$ \\
\hline Did you take the swab test? & 633 & $\begin{array}{l}\text { No } \\
\text { Yes }\end{array}$ & $\begin{array}{l}81 \%(51) \\
19 \%(12)\end{array}$ & $\begin{array}{l}96 \%(548) \\
4 \%(22)\end{array}$ & $\begin{array}{l}95 \%(599) \\
5 \%(34)\end{array}$ & $<0.001$ \\
\hline Have you lost anyone during this period? & 629 & $\begin{array}{l}\text { No } \\
\text { Yes }\end{array}$ & $\begin{array}{l}89 \%(56) \\
11 \%(7)\end{array}$ & $\begin{array}{l}90 \%(509) \\
10 \%(57)\end{array}$ & $\begin{array}{l}90 \%(565) \\
10 \%(64)\end{array}$ & 0.796 \\
\hline $\begin{array}{l}\text { Besides yourself, how many people live in } \\
\text { your home? }\end{array}$ & 1153 & $\begin{array}{l}\text { I do not live alone } \\
\text { I live alone }\end{array}$ & $\begin{array}{c}94 \%(59) \\
6 \%(4)\end{array}$ & $\begin{array}{l}85 \%(924) \\
15 \%(166)\end{array}$ & $\begin{array}{l}85 \%(983) \\
15 \%(170)\end{array}$ & 0.053 \\
\hline $\begin{array}{l}\text { AFTER the measures taken by the } \\
\text { government to contain the spread of the } \\
\text { coronavirus since you have been in } \\
\text { quarantine do you habitually practice some } \\
\text { physical activity }\end{array}$ & 967 & $\begin{array}{l}\text { No } \\
\text { Yes }\end{array}$ & $\begin{array}{l}73 \%(43) \\
27 \%(16)\end{array}$ & $\begin{array}{l}57 \%(516) \\
43 \%(392)\end{array}$ & $\begin{array}{l}58 \%(559) \\
42 \%(408)\end{array}$ & 0.016 \\
\hline Own a pet & 1178 & $\begin{array}{l}\text { No } \\
\text { Yes }\end{array}$ & $\begin{array}{l}33 \%(2 I) \\
67 \%(43)\end{array}$ & $\begin{array}{l}54 \%(606) \\
46 \%(508)\end{array}$ & $\begin{array}{l}53 \%(627) \\
47 \%(55 \mathrm{I})\end{array}$ & 0.001 \\
\hline
\end{tabular}

Note: Significant $P$ values between LEPRE and PRESTO groups are expressed in bold characters.

better situation from the point of view of psychological distress on both the GHQ and IES -R scales along with the depression scale CES-D, while cluster 2 is mainly composed of females reporting worse psychological distress on the GHQ-12 together with the IES-R scale and CES-D score. The two clusters are characterized by similar frequencies of people coming from the LEPRE and PRESTO cohorts indicating an overall homogeneity across the survey groups of responders (Table S8). Interestingly, independently from belonging to the LEPRE or PRESTO cohort, women showed the worst IES-R, GHQ-12, and CES-D scores (Table S8).

\section{Discussion}

Concerns, healthcare-related behaviors and psychological impact of COVID-19 pandemic among patients with rheumatic diseases and in particular with SLE have been investigated by several works, reporting a trend towards 
Table 2 Descriptive Statistics of the Survey Responders Post Propensity Score Matching

\begin{tabular}{|c|c|c|c|c|c|c|}
\hline Answer & $\begin{array}{c}\text { N. of } \\
\text { Responders }\end{array}$ & Variable & $\begin{array}{l}\text { LEPRE } \\
(\mathbf{N}=64)\end{array}$ & $\begin{array}{l}\text { PRESTO } \\
(\mathbf{N}=128)\end{array}$ & $\begin{array}{l}\text { Combined } \\
(N=192)\end{array}$ & $\begin{array}{c}\mathbf{P} \\
\text { value }\end{array}$ \\
\hline How old are you? & 192 & $\begin{array}{l}\text { Age, median (I- } \\
\text { III interquartile } \\
\text { range) }\end{array}$ & $43(35-54.5)$ & $46(39.5-55)$ & $45.5(37.8-55)$ & 0.467 \\
\hline Age class & 192 & $\begin{array}{c}<29 \\
29-65 \\
65+\end{array}$ & $\begin{array}{c}16 \%(10) \\
81 \%(52) \\
3 \%(2)\end{array}$ & $\begin{array}{c}9 \%(I 2) \\
88 \%(I I 2) \\
3 \%(4)\end{array}$ & $\begin{array}{c}11 \%(22) \\
85 \%(164) \\
3 \%(6)\end{array}$ & 0.438 \\
\hline What is your gender & 192 & $\begin{array}{l}\text { Female } \\
\text { Male }\end{array}$ & $\begin{array}{c}88 \%(56) \\
12 \%(8)\end{array}$ & $\begin{array}{c}85 \%(109) \\
15 \%(19)\end{array}$ & $\begin{array}{c}86 \%(165) \\
14 \%(27)\end{array}$ & 0.66 \\
\hline Please specify your nationality & 192 & $\begin{array}{l}\text { Other } \\
\text { Italian }\end{array}$ & $\begin{array}{c}3 \%(2) \\
97 \%(62)\end{array}$ & $\begin{array}{c}2 \%(2) \\
98 \%(126)\end{array}$ & $\begin{array}{c}2 \%(4) \\
98 \%(188)\end{array}$ & 0.475 \\
\hline What is your level of education? & 192 & $\begin{array}{c}\text { High School } \\
\text { University } \\
\text { Secondary school }\end{array}$ & $\begin{array}{c}70 \%(45) \\
28 \%(18) \\
2 \%(I)\end{array}$ & $\begin{array}{c}64 \%(82) \\
34 \%(44) \\
2 \%(2)\end{array}$ & $\begin{array}{c}66 \%(127) \\
32 \%(62) \\
2 \%(3)\end{array}$ & 0.681 \\
\hline What is your employment status? & 192 & $\begin{array}{l}\text { Employed } \\
\text { Student }\end{array}$ & $\begin{array}{c}97 \%(62) \\
3 \%(2)\end{array}$ & $\begin{array}{c}97 \%(124) \\
3 \%(4)\end{array}$ & $\begin{array}{c}97 \%(186) \\
3 \%(6)\end{array}$ & I \\
\hline What is your house like? & 192 & $\begin{array}{c}\text { Flat } \\
\text { Studio flat } \\
\text { Detached house }\end{array}$ & $\begin{array}{c}39 \%(25) \\
2 \%(1) \\
59 \%(38)\end{array}$ & $\begin{array}{c}38 \%(49) \\
1 \%(1) \\
61 \%(78)\end{array}$ & $\begin{array}{c}39 \%(74) \\
1 \%(2) \\
60 \%(116)\end{array}$ & 0.872 \\
\hline Can you access a private garden? & 192 & $\begin{array}{l}\text { No } \\
\text { Yes }\end{array}$ & $\begin{array}{l}19 \%(12) \\
81 \%(52)\end{array}$ & $\begin{array}{l}19 \%(24) \\
81 \%(104)\end{array}$ & $\begin{array}{l}19 \%(36) \\
81 \%(156)\end{array}$ & I \\
\hline Did you take the swab test? & 126 & $\begin{array}{l}\text { No } \\
\text { Yes }\end{array}$ & $\begin{array}{l}81 \%(51) \\
19 \%(12)\end{array}$ & $\begin{array}{c}86 \%(54) \\
14 \%(9)\end{array}$ & $\begin{array}{c}83 \%(105) \\
17 \%(21)\end{array}$ & 0.473 \\
\hline Have you lost anyone during this period? & 125 & $\begin{array}{l}\text { No } \\
\text { Yes }\end{array}$ & $\begin{array}{c}89 \%(56) \\
11 \%(7)\end{array}$ & $\begin{array}{c}94 \%(58) \\
6 \%(4)\end{array}$ & $\begin{array}{c}91 \%(114) \\
9 \%(I I)\end{array}$ & 0.358 \\
\hline $\begin{array}{l}\text { Besides yourself, how many people live in your } \\
\text { home? }\end{array}$ & 188 & $\begin{array}{l}\text { I do not live alone } \\
\text { I live alone }\end{array}$ & $\begin{array}{l}94 \%(59) \\
6 \%(4)\end{array}$ & $\begin{array}{l}94 \%(118) \\
6 \%(7)\end{array}$ & $\begin{array}{l}94 \%(177) \\
6 \%(11)\end{array}$ & 0.836 \\
\hline $\begin{array}{l}\text { AFTER the measures taken by the government to } \\
\text { contain the spread of the coronavirus since you } \\
\text { have been in quarantine do you habitually } \\
\text { practice some physical activity }\end{array}$ & 163 & $\begin{array}{l}\text { No } \\
\text { Yes }\end{array}$ & $\begin{array}{l}73 \%(43) \\
27 \%(16)\end{array}$ & $\begin{array}{l}73 \%(76) \\
27 \%(28)\end{array}$ & $\begin{array}{l}73 \%(119) \\
27 \%(44)\end{array}$ & 0.978 \\
\hline Own a pet & 192 & $\begin{array}{l}\text { No } \\
\text { Yes }\end{array}$ & $\begin{array}{l}33 \%(21) \\
67 \%(43)\end{array}$ & $\begin{array}{l}33 \%(42) \\
67 \%(86)\end{array}$ & $\begin{array}{l}33 \%(63) \\
67 \%(129)\end{array}$ & I \\
\hline $\begin{array}{l}\text { Mental health scores } \\
\text { GHQ-I2 Total score }\end{array}$ & 181 & $\begin{array}{l}0-13 \\
14-36\end{array}$ & $\begin{array}{l}15 \%(9) \\
85 \%(52)\end{array}$ & $\begin{array}{l}12 \%(14) \\
88 \%(106)\end{array}$ & $\begin{array}{l}13 \%(23) \\
87 \%(158)\end{array}$ & 0.556 \\
\hline IES-R Total score & 178 & $\begin{array}{l}0-23 \\
24-32 \\
33-36 \\
37-88\end{array}$ & $\begin{array}{l}43 \%(26) \\
27 \%(16) \\
7 \%(4) \\
23 \%(14)\end{array}$ & $\begin{array}{l}51 \%(60) \\
23 \%(27) \\
6 \%(7) \\
20 \%(24)\end{array}$ & $\begin{array}{l}48 \%(86) \\
24 \%(43) \\
6 \%(11) \\
21 \%(38)\end{array}$ & 0.825 \\
\hline CES-D Total score & 176 & $\begin{array}{l}0-15 \\
16-23 \\
24-60\end{array}$ & $\begin{array}{l}55 \%(33) \\
27 \%(16) \\
18 \%(11)\end{array}$ & $\begin{array}{l}60 \%(70) \\
22 \%(25) \\
18 \%(21)\end{array}$ & $\begin{array}{l}59 \%(103) \\
23 \%(41) \\
18 \%(32)\end{array}$ & 0.727 \\
\hline
\end{tabular}


Table 3 Descriptive Statistics of the Missing Activities ("Which of the Following Activities are You Missing the Most During This Quarantine Period?”) During the COVID-19 Quarantine (Post-Propensity Match Sample)

\begin{tabular}{|c|c|c|c|c|c|c|}
\hline Answer & $\begin{array}{c}\text { N. of } \\
\text { Responders }\end{array}$ & Response & $\begin{array}{l}\text { LEPRE } \\
(\mathbf{N}=64)\end{array}$ & $\begin{array}{l}\text { PRESTO } \\
(\mathbf{N}=128)\end{array}$ & $\begin{array}{l}\text { Combined } \\
(\mathrm{N}=192)\end{array}$ & $\begin{array}{c}P \\
\text { value }\end{array}$ \\
\hline Going to the cinema, theatre. & 191 & $\begin{array}{c}\text { Not selected } \\
\text { Yes }\end{array}$ & $\begin{array}{l}75 \%(47) \\
25 \%(16)\end{array}$ & $\begin{array}{l}73 \%(94) \\
27 \%(34)\end{array}$ & $\begin{array}{l}74 \%(14 I) \\
26 \%(50)\end{array}$ & 0.863 \\
\hline Playing sports/exercise. & 191 & $\begin{array}{c}\text { Not selected } \\
\text { Yes }\end{array}$ & $\begin{array}{l}81 \%(51) \\
19 \%(12)\end{array}$ & $\begin{array}{l}64 \%(82) \\
36 \%(46)\end{array}$ & $\begin{array}{l}70 \%(133) \\
30 \%(58)\end{array}$ & 0.017 \\
\hline $\begin{array}{l}\text { Going to exhibitions and } \\
\text { museums. }\end{array}$ & 191 & $\begin{array}{c}\text { Not selected } \\
\text { Yes }\end{array}$ & $\begin{array}{c}89 \%(56) \\
11 \%(7)\end{array}$ & $\begin{array}{l}88 \%(112) \\
12 \%(16)\end{array}$ & $\begin{array}{l}88 \%(168) \\
12 \%(23)\end{array}$ & 0.782 \\
\hline Going to nightclubs. & 191 & $\begin{array}{c}\text { Not selected } \\
\text { Yes }\end{array}$ & $\begin{array}{c}98 \%(62) \\
2 \%(1)\end{array}$ & $\begin{array}{c}98 \%(125) \\
2 \%(3)\end{array}$ & $\begin{array}{c}98 \%(187) \\
2 \%(4)\end{array}$ & 0.731 \\
\hline Attending sporting events. & 191 & $\begin{array}{c}\text { Not selected } \\
\text { Yes }\end{array}$ & $\begin{array}{c}95 \%(60) \\
5 \%(3)\end{array}$ & $\begin{array}{l}91 \%(117) \\
9 \%(11)\end{array}$ & $\begin{array}{c}93 \%(177) \\
7 \%(14)\end{array}$ & 0.339 \\
\hline Going out to eat. & 191 & $\begin{array}{c}\text { Not selected } \\
\text { Yes }\end{array}$ & $\begin{array}{l}56 \%(35) \\
44 \%(28)\end{array}$ & $\begin{array}{l}52 \%(66) \\
48 \%(62)\end{array}$ & $\begin{array}{l}53 \%(101) \\
47 \%(90)\end{array}$ & 0.603 \\
\hline Going to coffee shops. & 191 & $\begin{array}{c}\text { Not selected } \\
\text { Yes }\end{array}$ & $\begin{array}{l}84 \%(53) \\
16 \%(10)\end{array}$ & $\begin{array}{c}84 \%(107) \\
16 \%(21)\end{array}$ & $\begin{array}{l}84 \%(160) \\
16 \%(31)\end{array}$ & 0.925 \\
\hline Gardening. & 191 & $\begin{array}{c}\text { Not selected } \\
\text { Yes }\end{array}$ & $\begin{array}{c}95 \%(60) \\
5 \%(3)\end{array}$ & $\begin{array}{c}97 \%(124) \\
3 \%(4)\end{array}$ & $\begin{array}{c}96 \%(184) \\
4 \%(7)\end{array}$ & 0.571 \\
\hline Going out for a walk. & 191 & $\begin{array}{c}\text { Not selected } \\
\text { Yes }\end{array}$ & $\begin{array}{l}40 \%(25) \\
60 \%(38)\end{array}$ & $\begin{array}{l}38 \%(49) \\
62 \%(79)\end{array}$ & $\begin{array}{l}39 \%(74) \\
61 \%(117)\end{array}$ & 0.852 \\
\hline Going out with friends. & 191 & $\begin{array}{c}\text { Not selected } \\
\text { Yes }\end{array}$ & $\begin{array}{l}40 \%(25) \\
60 \%(38)\end{array}$ & $\begin{array}{l}41 \%(53) \\
59 \%(75)\end{array}$ & $\begin{array}{c}41 \%(78) \\
59 \%(I 13)\end{array}$ & 0.82 \\
\hline Seeing the family. & 191 & $\begin{array}{c}\text { Not selected } \\
\text { Yes }\end{array}$ & $\begin{array}{l}29 \%(18) \\
71 \%(45)\end{array}$ & $\begin{array}{l}67 \%(86) \\
33 \%(42)\end{array}$ & $\begin{array}{c}54 \%(104) \\
46 \%(87)\end{array}$ & $<0.001$ \\
\hline Going shopping. & 191 & $\begin{array}{c}\text { Not selected } \\
\text { Yes }\end{array}$ & $\begin{array}{l}78 \%(49) \\
22 \%(14)\end{array}$ & $\begin{array}{l}75 \%(96) \\
25 \%(32)\end{array}$ & $\begin{array}{c}76 \%(145) \\
24 \%(46)\end{array}$ & 0.673 \\
\hline Going to work. & 191 & $\begin{array}{c}\text { Not selected } \\
\text { Yes }\end{array}$ & $\begin{array}{l}75 \%(47) \\
25 \%(16)\end{array}$ & $\begin{array}{l}70 \%(89) \\
30 \%(39)\end{array}$ & $\begin{array}{l}71 \%(136) \\
29 \%(55)\end{array}$ & 0.467 \\
\hline
\end{tabular}

Note: Significant $P$ values between LEPRE and PRESTO groups are expressed in bold characters.

remarkable psychological distress, however with contrasting results; more specifically, the effect of restrictive measures among the same populations has been less analysed. $^{15-19}$

Our hypothesis, was that not only the fear of the infection but also the restriction-based strategy might upset the life of patients with SLE. To minimize attribution bias due to disease activity, we selected only SLE patients who were in remission or with low disease activity at the last follow-up visit, and we compared them to the general population, utilizing the same survey form pertaining to the same lockdown period. Globally, lockdown measures showed a great psychological impact on both patients and general population, half or more than half of both groups disclosing high scores of IES-R, GHQ-12 and CES-D questionnaires. However, our study showed no differences between SLE patients and the general population in IES-R, GHQ-12 and CES-D scores, while feeling sad or depressed was more common in the general population than in the patients, thus supporting the concept of resilience of SLE due to a previously adaptation to a difficult context (ie, chronic inflammatory disease). Patients did not show significant differences in their behavior if compared to the general population, except for two aspects: "missing playing sports or exercise" (less impact) and "missing to see the family" (more 
Table 4 Descriptive Statistics of the Responder's Feeling ("Since the Government Started the Restrictive Measures Since You are in Quarantine, What Do You Feel When You Think About the Pandemic and the Coronavirus?') During the COVID-19 Quarantine (PostPropensity Match Sample)

\begin{tabular}{|c|c|c|c|c|c|c|}
\hline Answer & $\begin{array}{c}\text { N. of } \\
\text { Responders }\end{array}$ & Response & $\begin{array}{l}\text { LEPRE } \\
(\mathbf{N}=64)\end{array}$ & $\begin{array}{l}\text { PRESTO } \\
(\mathrm{N}=128)\end{array}$ & $\begin{array}{l}\text { Combined } \\
(\mathrm{N}=192)\end{array}$ & $\begin{array}{c}P \\
\text { value }\end{array}$ \\
\hline $\begin{array}{l}\text { Do you have difficulty concentrating on what you do? Can you } \\
\text { follow the train of thought? Concentrate when reading, at } \\
\text { work, etc.? }\end{array}$ & 181 & $\begin{array}{l}\text { As usual } \\
\text { Less than usual } \\
\text { Much less than usual } \\
\text { More than usual }\end{array}$ & $\begin{array}{l}69 \%(42) \\
8 \%(5) \\
2 \%(1) \\
21 \%(13)\end{array}$ & $\begin{array}{l}57 \%(68) \\
18 \%(22) \\
7 \%(8) \\
18 \%(22)\end{array}$ & $\begin{array}{l}61 \%(110) \\
15 \%(27) \\
5 \%(9) \\
19 \%(35)\end{array}$ & 0.111 \\
\hline $\begin{array}{l}\text { Do you think you have lost a lot of sleep, enough to make you } \\
\text { worry? }\end{array}$ & 181 & $\begin{array}{l}\text { As usual } \\
\text { Less than usual } \\
\text { Much less than usual } \\
\text { More than usual }\end{array}$ & $\begin{array}{c}57 \%(35) \\
23 \%(14) \\
7 \%(4) \\
13 \%(8)\end{array}$ & $\begin{array}{l}52 \%(63) \\
13 \%(16) \\
16 \%(19) \\
18 \%(22)\end{array}$ & $\begin{array}{l}54 \%(98) \\
17 \%(30) \\
13 \%(23) \\
17 \%(30)\end{array}$ & 0.12 \\
\hline $\begin{array}{l}\text { Do you think you are productive, are you able to do many } \\
\text { things during the day? }\end{array}$ & 181 & $\begin{array}{l}\text { As usual } \\
\text { Less than usual } \\
\text { Much less than usual } \\
\text { More than usual }\end{array}$ & $\begin{array}{l}48 \%(29) \\
21 \%(13) \\
8 \%(5) \\
23 \%(14)\end{array}$ & $\begin{array}{l}38 \%(45) \\
29 \%(35) \\
11 \%(13) \\
22 \%(27)\end{array}$ & $\begin{array}{l}41 \%(74) \\
27 \%(48) \\
10 \%(18) \\
23 \%(4 I)\end{array}$ & 0.527 \\
\hline Do you think you are able to make decisions most of the time? & 181 & $\begin{array}{c}\text { As usual } \\
\text { Less than usual } \\
\text { Much less than usual } \\
\text { More than usual }\end{array}$ & $\begin{array}{c}77 \%(47) \\
16 \%(10) \\
0 \%(0) \\
7 \%(4)\end{array}$ & $\begin{array}{c}81 \%(97) \\
13 \%(16) \\
1 \%(1) \\
5 \%(6)\end{array}$ & $\begin{array}{c}80 \%(144) \\
14 \%(26) \\
1 \%(1) \\
6 \%(10)\end{array}$ & 0.796 \\
\hline Do you feel constantly under pressure? & 181 & $\begin{array}{l}\text { As usual } \\
\text { Less than usual } \\
\text { Much less than usual } \\
\text { More than usual }\end{array}$ & $\begin{array}{c}33 \%(20) \\
10 \%(6) \\
10 \%(6) \\
48 \%(29)\end{array}$ & $\begin{array}{l}34 \%(4 I) \\
18 \%(2 I) \\
8 \%(10) \\
40 \%(48)\end{array}$ & $\begin{array}{l}34 \%(61) \\
15 \%(27) \\
9 \%(16) \\
43 \%(77)\end{array}$ & 0.52 \\
\hline Do you think you are able to overcome difficulties right now? & 181 & $\begin{array}{c}\text { As usual } \\
\text { Less than usual } \\
\text { Much less than usual } \\
\text { More than usual }\end{array}$ & $\begin{array}{c}52 \%(32) \\
15 \%(9) \\
2 \%(1) \\
31 \%(19)\end{array}$ & $\begin{array}{c}56 \%(67) \\
15 \%(18) \\
2 \%(2) \\
28 \%(33)\end{array}$ & $\begin{array}{c}55 \%(99) \\
15 \%(27) \\
2 \%(3) \\
29 \%(52)\end{array}$ & 0.965 \\
\hline $\begin{array}{l}\text { During this period, are you able to find some free time and } \\
\text { enjoy it? }\end{array}$ & 181 & $\begin{array}{l}\text { As usual } \\
\text { Less than usual } \\
\text { Much less than usual } \\
\text { More than usual }\end{array}$ & $\begin{array}{c}46 \%(28) \\
20 \%(12) \\
13 \%(8) \\
21 \%(13)\end{array}$ & $\begin{array}{c}25 \%(30) \\
27 \%(32) \\
9 \%(11) \\
39 \%(47)\end{array}$ & $\begin{array}{l}32 \%(58) \\
24 \%(44) \\
10 \%(19) \\
33 \%(60)\end{array}$ & 0.013 \\
\hline In this situation, do you feel able to solve your problems? & 181 & $\begin{array}{l}\text { As usual } \\
\text { Less than usual } \\
\text { Much less than usual } \\
\text { More than usual }\end{array}$ & $\begin{array}{c}77 \%(47) \\
18 \%(11) \\
5 \%(3) \\
0 \%(0)\end{array}$ & $\begin{array}{c}59 \%(71) \\
24 \%(29) \\
6 \%(7) \\
11 \%(13)\end{array}$ & $\begin{array}{c}65 \%(118) \\
22 \%(40) \\
6 \%(10) \\
7 \%(13)\end{array}$ & 0.025 \\
\hline Sad or depressed. & 181 & $\begin{array}{l}\text { As usual } \\
\text { Less than usual } \\
\text { Much less than usual } \\
\text { More than usual }\end{array}$ & $\begin{array}{c}38 \%(23) \\
18 \%(11) \\
10 \%(6) \\
34 \%(21)\end{array}$ & $\begin{array}{c}46 \%(55) \\
5 \%(6) \\
6 \%(7) \\
43 \%(52)\end{array}$ & $\begin{array}{l}43 \%(78) \\
9 \%(17) \\
7 \%(13) \\
40 \%(73)\end{array}$ & 0.022 \\
\hline As if I have lost confidence in myself. & 181 & $\begin{array}{l}\text { As usual } \\
\text { Less than usual } \\
\text { Much less than usual } \\
\text { More than usual }\end{array}$ & $\begin{array}{c}54 \%(33) \\
15 \%(9) \\
18 \%(11) \\
13 \%(8)\end{array}$ & $\begin{array}{l}68 \%(82) \\
9 \%(11) \\
9 \%(11) \\
13 \%(16)\end{array}$ & $\begin{array}{l}64 \%(115) \\
11 \%(20) \\
12 \%(22) \\
13 \%(24)\end{array}$ & 0.168 \\
\hline As if I had less self-esteem. & 181 & $\begin{array}{l}\text { As usual } \\
\text { Less than usual } \\
\text { Much less than usual } \\
\text { More than usual }\end{array}$ & $\begin{array}{c}57 \%(35) \\
16 \%(10) \\
16 \%(10) \\
10 \%(6)\end{array}$ & $\begin{array}{l}74 \%(89) \\
8 \%(10) \\
8 \%(10) \\
9 \%(11)\end{array}$ & $\begin{array}{c}69 \%(124) \\
11 \%(20) \\
11 \%(20) \\
9 \%(17)\end{array}$ & 0.092 \\
\hline
\end{tabular}


Table 4 (Continued).

\begin{tabular}{|c|c|c|c|c|c|c|}
\hline Answer & $\begin{array}{c}\text { N. of } \\
\text { Responders }\end{array}$ & Response & $\begin{array}{l}\text { LEPRE } \\
(\mathrm{N}=64)\end{array}$ & $\begin{array}{l}\text { PRESTO } \\
(\mathrm{N}=\mid \mathbf{2 8})\end{array}$ & $\begin{array}{c}\text { Combined } \\
(\mathrm{N}=192)\end{array}$ & $\begin{array}{c}P \\
\text { value }\end{array}$ \\
\hline \multirow[t]{4}{*}{ With a overall happy emotional state. } & \multirow[t]{4}{*}{181} & As usual & $51 \%(3 I)$ & $45 \%(54)$ & $47 \%(85)$ & \multirow[t]{4}{*}{0.392} \\
\hline & & Less than usual & $28 \%(17)$ & $34 \%(4 I)$ & $32 \%(58)$ & \\
\hline & & Much less than usual & $7 \%(4)$ & $12 \%(14)$ & $10 \%(18)$ & \\
\hline & & More than usual & $15 \%(9)$ & $9 \%(11)$ & $11 \%(20)$ & \\
\hline \multirow{5}{*}{$\begin{array}{l}\text { Everything that reminds me of the pandemic makes me feel } \\
\text { emotions. }\end{array}$} & \multirow[t]{5}{*}{178} & Quite a bit & $32 \%(19)$ & $35 \%(4 I)$ & $34 \%(60)$ & \multirow[t]{5}{*}{0.589} \\
\hline & & Extremely & $17 \%(10)$ & $8 \%(10)$ & II\% (20) & \\
\hline & & Moderately & $18 \%(I I)$ & $21 \%(25)$ & $20 \%(36)$ & \\
\hline & & Not at all & $7 \%(4)$ & $8 \%(10)$ & $8 \%(14)$ & \\
\hline & & A little bit & $27 \%(16)$ & $27 \%(32)$ & $27 \%(48)$ & \\
\hline \multirow[t]{5}{*}{ I had difficulty staying asleep. } & \multirow[t]{5}{*}{178} & Quite a bit & $15 \%(9)$ & $15 \%(18)$ & $15 \%(27)$ & \multirow[t]{5}{*}{0.806} \\
\hline & & Extremely & $5 \%(3)$ & $2 \%(2)$ & $3 \%(5)$ & \\
\hline & & Moderately & $12 \%(7)$ & $13 \%(15)$ & $12 \%(22)$ & \\
\hline & & Not at all & $43 \%(26)$ & $44 \%$ (52) & $44 \%(78)$ & \\
\hline & & A little bit & $25 \%(15)$ & $26 \%(3 I)$ & $26 \%(46)$ & \\
\hline \multirow{5}{*}{$\begin{array}{l}\text { Things that do not concern the pandemic make me think about } \\
\text { it anyway. }\end{array}$} & \multirow[t]{5}{*}{178} & Quite a bit & $18 \%(11)$ & $16 \%(19)$ & $17 \%(30)$ & \multirow[t]{5}{*}{0.907} \\
\hline & & Extremely & $3 \%(2)$ & $2 \%(2)$ & $2 \%(4)$ & \\
\hline & & Moderately & $20 \%(12)$ & $20 \%(24)$ & $20 \%(36)$ & \\
\hline & & Not at all & $27 \%(16)$ & $25 \%(29)$ & $25 \%(45)$ & \\
\hline & & A little bit & $32 \%(19)$ & $37 \%(44)$ & $35 \%(63)$ & \\
\hline \multirow[t]{5}{*}{ I feel irritable and angry. } & \multirow[t]{5}{*}{178} & Quite a bit & $15 \%(9)$ & $19 \%(22)$ & $17 \%(3 \mid)$ & \multirow[t]{5}{*}{0.221} \\
\hline & & Extremely & $10 \%(6)$ & $3 \%(3)$ & $5 \%(9)$ & \\
\hline & & Moderately & $22 \%(13)$ & $20 \%(24)$ & $21 \%(37)$ & \\
\hline & & Not at all & $23 \%(14)$ & $31 \%(37)$ & $29 \%(5 \mathrm{I})$ & \\
\hline & & A little bit & $30 \%(18)$ & $27 \%(32)$ & $28 \%(50)$ & \\
\hline \multirow{5}{*}{$\begin{array}{l}\text { I avoid getting upset when I think about it or it is reminded me } \\
\text { by others. }\end{array}$} & \multirow[t]{5}{*}{178} & Quite a bit & $17 \%(10)$ & $21 \%(25)$ & $20 \%(35)$ & \multirow[t]{5}{*}{0.844} \\
\hline & & Extremely & $7 \%(4)$ & $5 \%(6)$ & $6 \%(10)$ & \\
\hline & & Moderately & $27 \%(16)$ & $28 \%(33)$ & $28 \%(49)$ & \\
\hline & & Not at all & $20 \%(12)$ & $14 \%(17)$ & $16 \%(29)$ & \\
\hline & & A little bit & $30 \%(18)$ & $31 \%(37)$ & $31 \%(55)$ & \\
\hline \multirow[t]{5}{*}{ I think about it without intending to. } & \multirow[t]{5}{*}{178} & Quite a bit & $20 \%(12)$ & $10 \%(12)$ & $13 \%(24)$ & \multirow[t]{5}{*}{0.16} \\
\hline & & Extremely & $3 \%(2)$ & $4 \%(5)$ & $4 \%(7)$ & \\
\hline & & Moderately & $28 \%$ (17) & $21 \%(25)$ & $24 \%(42)$ & \\
\hline & & Not at all & $20 \%(12)$ & $19 \%(23)$ & $20 \%(35)$ & \\
\hline & & A little bit & $28 \%(17)$ & $45 \%(53)$ & $39 \%(70)$ & \\
\hline \multirow[t]{5}{*}{ I have a feeling it did not happen or it is not real. } & \multirow[t]{5}{*}{178} & Quite a bit & $13 \%(8)$ & $10 \%(12)$ & $11 \%(20)$ & 0.187 \\
\hline & & Extremely & $0 \%(0)$ & $2 \%(2)$ & $1 \%(2)$ & \\
\hline & & Moderately & $12 \%(7)$ & $17 \%(20)$ & $15 \%(27)$ & \\
\hline & & Not at all & $60 \%(36)$ & $45 \%(53)$ & $50 \%(89)$ & \\
\hline & & A little bit & $15 \%(9)$ & $26 \%(3 I)$ & $22 \%(40)$ & \\
\hline I avoided things that could remind it to me. & 178 & Quite a bit & $3 \%(2)$ & $8 \%(10)$ & $7 \%(12)$ & 0.029 \\
\hline & & Extremely & $3 \%(2)$ & $1 \%(1)$ & $2 \%(3)$ & \\
\hline & & Moderately & $17 \%(10)$ & $12 \%(14)$ & $13 \%(24)$ & \\
\hline & & Not at all & $37 \%(22)$ & $56 \%(66)$ & $49 \%(88)$ & \\
\hline & & A little bit & $40 \%$ (24) & $23 \%(27)$ & $29 \%(5 I)$ & \\
\hline
\end{tabular}

(Continued) 
Table 4 (Continued).

\begin{tabular}{|c|c|c|c|c|c|c|}
\hline Answer & $\begin{array}{c}\text { N. of } \\
\text { Responders }\end{array}$ & Response & $\begin{array}{l}\text { LEPRE } \\
(\mathrm{N}=64)\end{array}$ & $\begin{array}{l}\text { PRESTO } \\
(\mathrm{N}=128)\end{array}$ & $\begin{array}{l}\text { Combined } \\
(\mathrm{N}=192)\end{array}$ & $\begin{array}{c}P \\
\text { value }\end{array}$ \\
\hline $\begin{array}{l}\text { Some images about the pandemic, coronavirus, come to mind } \\
\text { suddenly. }\end{array}$ & 178 & $\begin{array}{l}\text { Quite a bit } \\
\text { Extremely } \\
\text { Moderately } \\
\text { Not at all } \\
\text { A little bit }\end{array}$ & $\begin{array}{c}5 \%(3) \\
3 \%(2) \\
23 \%(14) \\
35 \%(2 I) \\
33 \%(20)\end{array}$ & $\begin{array}{c}6 \%(7) \\
3 \%(4) \\
14 \%(16) \\
49 \%(58) \\
28 \%(33)\end{array}$ & $\begin{array}{c}6 \%(10) \\
3 \%(6) \\
17 \%(30) \\
44 \%(79) \\
30 \%(53)\end{array}$ & 0.343 \\
\hline I am nervous and easily scared. & 178 & $\begin{array}{l}\text { Quite a bit } \\
\text { Extremely } \\
\text { Moderately } \\
\text { Not at all } \\
\text { A little bit }\end{array}$ & $\begin{array}{c}13 \%(8) \\
3 \%(2) \\
15 \%(9) \\
37 \%(22) \\
32 \%(19)\end{array}$ & $\begin{array}{c}9 \%(11) \\
4 \%(5) \\
11 \%(13) \\
44 \%(52) \\
31 \%(37)\end{array}$ & $\begin{array}{c}11 \%(19) \\
4 \%(7) \\
12 \%(22) \\
42 \%(74) \\
31 \%(56)\end{array}$ & 0.787 \\
\hline I try not to think about it. & 178 & $\begin{array}{l}\text { Quite a bit } \\
\text { Extremely } \\
\text { Moderately } \\
\text { Not at all } \\
\text { A little bit }\end{array}$ & $\begin{array}{c}20 \%(12) \\
10 \%(6) \\
22 \%(13) \\
17 \%(10) \\
32 \%(19)\end{array}$ & $\begin{array}{c}21 \%(25) \\
3 \%(4) \\
19 \%(23) \\
20 \%(24) \\
36 \%(42)\end{array}$ & $\begin{array}{c}21 \%(37) \\
6 \%(10) \\
20 \%(36) \\
19 \%(34) \\
34 \%(61)\end{array}$ & 0.452 \\
\hline $\begin{array}{l}\text { I am aware that I still can not manage all my emotions regarding } \\
\text { the pandemic, coronavirus. }\end{array}$ & 178 & $\begin{array}{l}\text { Quite a bit } \\
\text { Extremely } \\
\text { Moderately } \\
\text { Not at all } \\
\text { A little bit }\end{array}$ & $\begin{array}{c}18 \%(1 \mid) \\
3 \%(2) \\
22 \%(13) \\
28 \%(17) \\
28 \%(17)\end{array}$ & $\begin{array}{c}12 \%(\mid 4) \\
4 \%(5) \\
23 \%(27) \\
31 \%(36) \\
31 \%(36)\end{array}$ & $\begin{array}{c}14 \%(25) \\
4 \%(7) \\
22 \%(40) \\
30 \%(53) \\
30 \%(53)\end{array}$ & 0.841 \\
\hline My feelings about it are kind of numb. & 178 & $\begin{array}{l}\text { Quite a bit } \\
\text { Extremely } \\
\text { Moderately } \\
\text { Not at all } \\
\text { A little bit }\end{array}$ & $\begin{array}{c}7 \%(4) \\
0 \%(0) \\
10 \%(6) \\
58 \%(35) \\
25 \%(15)\end{array}$ & $\begin{array}{c}8 \%(9) \\
3 \%(3) \\
16 \%(19) \\
52 \%(61) \\
22 \%(26)\end{array}$ & $\begin{array}{c}7 \%(13) \\
2 \%(3) \\
14 \%(25) \\
54 \%(96) \\
23 \%(4 I)\end{array}$ & 0.54 \\
\hline $\begin{array}{l}\text { I found myself acting or feeling as though I was back at the time } \\
\text { when the quarantine was announced. }\end{array}$ & 178 & $\begin{array}{l}\text { Quite a bit } \\
\text { Extremely } \\
\text { Moderately } \\
\text { Not at all } \\
\text { A little bit }\end{array}$ & $\begin{array}{c}7 \%(4) \\
0 \%(0) \\
20 \%(12) \\
48 \%(29) \\
25 \%(15)\end{array}$ & $\begin{array}{c}4 \%(5) \\
0 \%(0) \\
16 \%(19) \\
60 \%(7 I) \\
19 \%(23)\end{array}$ & $\begin{array}{c}5 \%(9) \\
0 \%(0) \\
17 \%(31) \\
56 \%(100) \\
21 \%(38)\end{array}$ & 0.5 \\
\hline I have trouble falling asleep. & 178 & $\begin{array}{l}\text { Quite a bit } \\
\text { Extremely } \\
\text { Moderately } \\
\text { Not at all } \\
\text { A little bit }\end{array}$ & $\begin{array}{c}7 \%(4) \\
8 \%(5) \\
12 \%(7) \\
43 \%(26) \\
30 \%(18)\end{array}$ & $\begin{array}{c}9 \%(11) \\
4 \%(5) \\
9 \%(11) \\
58 \%(69) \\
19 \%(22)\end{array}$ & $\begin{array}{c}8 \%(15) \\
6 \%(10) \\
10 \%(18) \\
53 \%(95) \\
22 \%(40)\end{array}$ & 0.22 \\
\hline I have waves of strong feelings when I think about it. & 178 & $\begin{array}{l}\text { Quite a bit } \\
\text { Extremely } \\
\text { Moderately } \\
\text { Not at all } \\
\text { A little bit }\end{array}$ & $\begin{array}{c}18 \%(1 \mathrm{I}) \\
3 \%(2) \\
27 \%(16) \\
18 \%(11) \\
33 \%(20)\end{array}$ & $\begin{array}{c}15 \%(18) \\
6 \%(7) \\
19 \%(23) \\
28 \%(33) \\
31 \%(37)\end{array}$ & $\begin{array}{c}16 \%(29) \\
5 \%(9) \\
22 \%(39) \\
25 \%(44) \\
32 \%(57)\end{array}$ & 0.519 \\
\hline I tried to remove it from my memory. & 178 & $\begin{array}{l}\text { Quite a bit } \\
\text { Extremely } \\
\text { Moderately } \\
\text { Not at all } \\
\text { A little bit }\end{array}$ & $\begin{array}{c}13 \%(8) \\
2 \%(1) \\
20 \%(12) \\
43 \%(26) \\
22 \%(13)\end{array}$ & $\begin{array}{c}11 \%(13) \\
7 \%(8) \\
19 \%(22) \\
42 \%(50) \\
21 \%(25)\end{array}$ & $\begin{array}{c}12 \%(2 \mathrm{I}) \\
5 \%(9) \\
19 \%(34) \\
43 \%(76) \\
21 \%(38)\end{array}$ & 0.683 \\
\hline
\end{tabular}

(Continued) 
Table 4 (Continued).

\begin{tabular}{|c|c|c|c|c|c|c|}
\hline Answer & $\begin{array}{c}\text { N. of } \\
\text { Responders }\end{array}$ & Response & $\begin{array}{l}\text { LEPRE } \\
(\mathrm{N}=64)\end{array}$ & $\begin{array}{l}\text { PRESTO } \\
(\mathrm{N}=128)\end{array}$ & $\begin{array}{l}\text { Combined } \\
(\mathrm{N}=192)\end{array}$ & $\begin{array}{c}P \\
\text { value }\end{array}$ \\
\hline I have trouble concentrating. & 178 & $\begin{array}{l}\text { Quite a bit } \\
\text { Extremely } \\
\text { Moderately } \\
\text { Not at all } \\
\text { A little bit }\end{array}$ & $\begin{array}{c}12 \%(7) \\
3 \%(2) \\
10 \%(6) \\
45 \%(27) \\
30 \%(18)\end{array}$ & $\begin{array}{c}8 \%(10) \\
4 \%(5) \\
8 \%(10) \\
52 \%(61) \\
27 \%(32)\end{array}$ & $\begin{array}{c}10 \%(17) \\
4 \%(7) \\
9 \%(16) \\
49 \%(88) \\
28 \%(50)\end{array}$ & 0.896 \\
\hline $\begin{array}{l}\text { Reminders of it cause me to have physical reactions, such as } \\
\text { sweating, trouble breathing, nausea, or a pounding heart. }\end{array}$ & 178 & $\begin{array}{l}\text { Quite a bit } \\
\text { Extremely } \\
\text { Moderately } \\
\text { Not at all } \\
\text { A little bit }\end{array}$ & $\begin{array}{c}7 \%(4) \\
3 \%(2) \\
5 \%(3) \\
67 \%(40) \\
18 \%(11)\end{array}$ & $\begin{array}{c}3 \%(3) \\
2 \%(2) \\
4 \%(5) \\
75 \%(89) \\
16 \%(19)\end{array}$ & $\begin{array}{c}4 \%(7) \\
2 \%(4) \\
4 \%(8) \\
72 \%(129) \\
17 \%(30)\end{array}$ & 0.594 \\
\hline I have dreams about the pandemic/coronavirus. & 178 & $\begin{array}{l}\text { Quite a bit } \\
\text { Extremely } \\
\text { Moderately } \\
\text { Not at all } \\
\text { A little bit }\end{array}$ & $\begin{array}{c}5 \%(3) \\
3 \%(2) \\
2 \%(1) \\
75 \%(45) \\
15 \%(9)\end{array}$ & $\begin{array}{c}2 \%(2) \\
2 \%(2) \\
4 \%(5) \\
78 \%(92) \\
14 \%(17)\end{array}$ & $\begin{array}{c}3 \%(5) \\
2 \%(4) \\
3 \%(6) \\
77 \%(137) \\
15 \%(26)\end{array}$ & 0.582 \\
\hline $\begin{array}{l}\text { I have become watchful and on-guard with people and the } \\
\text { environment. }\end{array}$ & 178 & $\begin{array}{l}\text { Quite a bit } \\
\text { Extremely } \\
\text { Moderately } \\
\text { Not at all } \\
\text { A little bit }\end{array}$ & $\begin{array}{c}27 \%(16) \\
15 \%(9) \\
25 \%(15) \\
15 \%(9) \\
18 \%(11)\end{array}$ & $\begin{array}{c}18 \%(2 I) \\
7 \%(8) \\
24 \%(28) \\
21 \%(25) \\
31 \%(36)\end{array}$ & $\begin{array}{l}21 \%(37) \\
10 \%(17) \\
24 \%(43) \\
19 \%(34) \\
26 \%(47)\end{array}$ & 0.117 \\
\hline I try not to talk about the pandemic/coronavirus. & 178 & $\begin{array}{l}\text { Quite a bit } \\
\text { Extremely } \\
\text { Moderately } \\
\text { Not at all } \\
\text { A little bit }\end{array}$ & $\begin{array}{c}7 \%(4) \\
2 \%(1) \\
17 \%(10) \\
50 \%(30) \\
25 \%(15)\end{array}$ & $\begin{array}{c}5 \%(6) \\
2 \%(2) \\
26 \%(3 I) \\
37 \%(44) \\
30 \%(35)\end{array}$ & $\begin{array}{c}6 \%(10) \\
2 \%(3) \\
23 \%(41) \\
42 \%(74) \\
28 \%(50)\end{array}$ & 0.459 \\
\hline
\end{tabular}

Note: Significant $P$ values between LEPRE and PRESTO groups are expressed in bold characters.

impact). No difference to the general population was observed about anxiety or depression rate, self-esteem, confidence in the future, sleep disorders, appetite, or even food consumption. On the other hand, the patients could be more able to cope with the problem of the outbreak, also by "avoiding things that could remind it", that refers to a previously adopted coping strategy. Interestingly, the patients' lifestyle (eg, living in detached houses, having access to a private garden and also owning a pet) could contribute to cope with restrictions. Notably, this coping strategy might be of value also for healthy people, in order to limit the psychological distress of restrictive measures and to mitigate the risk of mental disorders. Indeed, the possibility of communicating with medical staff can affect patients' ability to adapt to the stressful situation caused by the pandemic. ${ }^{20}$ Finally, patients consumed fewer eggs than general population, adhering more tightly to the Mediterranean diet. Thus, resilience of patients with SLE probably rests on three pillars: family and relatives, lifestyle and access to care (Figure 1).

Limits of our study were the lack of knowledge of the pre-pandemic lifestyle of the enrolled patients and the anonymous nature of the questionnaire, that did not allow us to perform clinical associations. Also, the correct inclusion of PRESTO responders in the two categories (with or without chronic disease) could not be verified by a doctor due to the self-administered nature of the questionnaire. On the other hand, the high number of participants in the PRESTO study and the reproducibility of the results in all the three sub analyses, after the propensity score matching procedures, resulted in a robust comparison between patients and general population minimizing potential selection bias. Also, generalizability of the conclusion is limited, since the inclusion criteria comprised remission or low disease activity state, while 
Table 5 Descriptive Statistics of the Responder's Emotional Status During the COVID-19 Quarantine (Post-Propensity Match Sample) ("the Following Questions Refer to the Quarantine Period")

\begin{tabular}{|c|c|c|c|c|c|c|}
\hline Answer & $\begin{array}{c}\text { N. of } \\
\text { Responders }\end{array}$ & Response & $\begin{array}{l}\text { LEPRE } \\
(\mathrm{N}=64)\end{array}$ & $\begin{array}{l}\text { PRESTO } \\
(N=\mid 28)\end{array}$ & $\begin{array}{l}\text { Combined } \\
(\mathrm{N}=192)\end{array}$ & $\begin{array}{c}P \\
\text { value }\end{array}$ \\
\hline $\begin{array}{l}\text { I have been hindered by things } \\
\text { that do not usually bother me. }\end{array}$ & 176 & $\begin{array}{l}\text { Frequently, all the time ( } 5 \text { to } 7 \text { days) } \\
\text { Never/Very rarely (less than a day) } \\
\text { Very often ( } 3 \text { to } 4 \text { days) } \\
\text { Occasionally (I to } 2 \text { days) }\end{array}$ & $\begin{array}{c}0 \%(0) \\
47 \%(28) \\
13 \%(8) \\
40 \%(24)\end{array}$ & $\begin{array}{l}3 \%(4) \\
60 \%(70) \\
8 \%(9) \\
28 \%(33)\end{array}$ & $\begin{array}{c}2 \%(4) \\
56 \%(98) \\
10 \%(17) \\
32 \%(57)\end{array}$ & 0.098 \\
\hline $\begin{array}{l}\text { I had no desire to eat nor } \\
\text { appetite. }\end{array}$ & 176 & $\begin{array}{l}\text { Frequently, all the time ( } 5 \text { to } 7 \text { days) } \\
\text { Never/Very rarely (less than a day) } \\
\text { Very often ( } 3 \text { to } 4 \text { days) } \\
\text { Occasionally ( } I \text { to } 2 \text { days) }\end{array}$ & $\begin{array}{c}2 \%(1) \\
83 \%(50) \\
2 \%(1) \\
13 \%(8)\end{array}$ & $\begin{array}{c}3 \%(3) \\
83 \%(96) \\
5 \%(6) \\
9 \%(11)\end{array}$ & $\begin{array}{c}2 \%(4) \\
83 \%(146) \\
4 \%(7) \\
11 \%(19)\end{array}$ & 0.59 \\
\hline $\begin{array}{l}\text { I got the impression that I could } \\
\text { not get out of depression. }\end{array}$ & 176 & $\begin{array}{l}\text { Frequently, all the time ( } 5 \text { to } 7 \text { days) } \\
\text { Never/Very rarely (less than a day) } \\
\text { Very often ( } 3 \text { to } 4 \text { days) } \\
\text { Occasionally ( } I \text { to } 2 \text { days) }\end{array}$ & $\begin{array}{c}0 \%(0) \\
85 \%(5 \mathrm{I}) \\
3 \%(2) \\
12 \%(7)\end{array}$ & $\begin{array}{c}1 \%(1) \\
82 \%(95) \\
4 \%(5) \\
13 \%(15)\end{array}$ & $\begin{array}{c}1 \%(1) \\
83 \%(146) \\
4 \%(7) \\
12 \%(22)\end{array}$ & $0.87 I$ \\
\hline $\begin{array}{l}\text { I had the feeling of being different } \\
\text { from others. }\end{array}$ & 176 & $\begin{array}{l}\text { Frequently, all the time ( } 5 \text { to } 7 \text { days) } \\
\text { Never/Very rarely (less than a day) } \\
\text { Very often ( } 3 \text { to } 4 \text { days) } \\
\text { Occasionally (I to } 2 \text { days) }\end{array}$ & $\begin{array}{c}5 \%(3) \\
50 \%(30) \\
23 \%(14) \\
22 \%(13)\end{array}$ & $\begin{array}{l}3 \%(4) \\
67 \%(78) \\
10 \%(12) \\
19 \%(22)\end{array}$ & $\begin{array}{c}4 \%(7) \\
61 \%(108) \\
15 \%(26) \\
20 \%(35)\end{array}$ & 0.078 \\
\hline $\begin{array}{l}\text { I had trouble concentrating on } \\
\text { what I was doing. }\end{array}$ & 176 & $\begin{array}{l}\text { Frequently, all the time ( } 5 \text { to } 7 \text { days) } \\
\text { Never/Very rarely (less than a day) } \\
\text { Very often ( } 3 \text { to } 4 \text { days) } \\
\text { Occasionally ( } I \text { to } 2 \text { days) }\end{array}$ & $\begin{array}{c}0 \%(0) \\
55 \%(33) \\
12 \%(7) \\
33 \%(20)\end{array}$ & $\begin{array}{l}9 \%(10) \\
54 \%(63) \\
9 \%(10) \\
28 \%(33)\end{array}$ & $\begin{array}{l}6 \%(10) \\
55 \%(96) \\
10 \%(17) \\
30 \%(53)\end{array}$ & 0.118 \\
\hline I felt depressed. & 176 & $\begin{array}{l}\text { Frequently, all the time ( } 5 \text { to } 7 \text { days) } \\
\text { Never/Very rarely (less than a day) } \\
\text { Very often ( } 3 \text { to } 4 \text { days) } \\
\text { Occasionally (I to } 2 \text { days) }\end{array}$ & $\begin{array}{c}2 \%(1) \\
62 \%(37) \\
8 \%(5) \\
28 \%(17)\end{array}$ & $\begin{array}{l}4 \%(5) \\
59 \%(69) \\
13 \%(15) \\
23 \%(27)\end{array}$ & $\begin{array}{c}3 \%(6) \\
60 \%(106) \\
11 \%(20) \\
25 \%(44)\end{array}$ & 0.576 \\
\hline $\begin{array}{l}\text { I got the impression that any } \\
\text { action required a lot of effort. }\end{array}$ & 176 & $\begin{array}{l}\text { Frequently, all the time ( } 5 \text { to } 7 \text { days) } \\
\text { Never/Very rarely (less than a day) } \\
\text { Very often ( } 3 \text { to } 4 \text { days) } \\
\text { Occasionally (I to } 2 \text { days) }\end{array}$ & $\begin{array}{l}2 \%(1) \\
53 \%(32) \\
17 \%(10) \\
28 \%(17)\end{array}$ & $\begin{array}{c}6 \%(7) \\
60 \%(70) \\
15 \%(17) \\
19 \%(22)\end{array}$ & $\begin{array}{c}5 \%(8) \\
58 \%(102) \\
15 \%(27) \\
22 \%(39)\end{array}$ & 0.3 \\
\hline I have faith in the future. & 176 & $\begin{array}{l}\text { Frequently, all the time ( } 5 \text { to } 7 \text { days) } \\
\text { Never/Very rarely (less than a day) } \\
\text { Very often ( } 3 \text { to } 4 \text { days) } \\
\text { Occasionally ( } I \text { to } 2 \text { days) }\end{array}$ & $\begin{array}{l}13 \%(8) \\
13 \%(8) \\
42 \%(25) \\
32 \%(19)\end{array}$ & $\begin{array}{l}28 \%(32) \\
9 \%(I I) \\
35 \%(4 I) \\
28 \%(32)\end{array}$ & $\begin{array}{l}23 \%(40) \\
11 \%(19) \\
38 \%(66) \\
29 \%(51)\end{array}$ & 0.193 \\
\hline I thought my life was a failure. & 176 & $\begin{array}{c}\text { Frequently, all the time ( } 5 \text { to } 7 \text { days) } \\
\text { Never/Very rarely (less than a day) } \\
\text { Very often ( } 3 \text { to } 4 \text { days) } \\
\text { Occasionally ( } I \text { to } 2 \text { days) }\end{array}$ & $\begin{array}{l}0 \%(0) \\
78 \%(47) \\
8 \%(5) \\
13 \%(8)\end{array}$ & $\begin{array}{c}3 \%(3) \\
74 \%(86) \\
9 \%(10) \\
15 \%(17)\end{array}$ & $\begin{array}{l}2 \%(3) \\
76 \%(133) \\
9 \%(15) \\
14 \%(25)\end{array}$ & 0.638 \\
\hline I felt scared. & 176 & $\begin{array}{l}\text { Frequently, all the time ( } 5 \text { to } 7 \text { days) } \\
\text { Never/Very rarely (less than a day) } \\
\text { Very often ( } 3 \text { to } 4 \text { days) } \\
\text { Occasionally ( } I \text { to } 2 \text { days) }\end{array}$ & $\begin{array}{c}10 \%(6) \\
32 \%(19) \\
28 \%(17) \\
30 \%(18)\end{array}$ & $\begin{array}{c}3 \%(3) \\
33 \%(38) \\
22 \%(25) \\
43 \%(50)\end{array}$ & $\begin{array}{c}5 \%(9) \\
32 \%(57) \\
24 \%(42) \\
39 \%(68)\end{array}$ & 0.079 \\
\hline I can not sleep well. & 176 & $\begin{array}{c}\text { Frequently, all the time ( } 5 \text { to } 7 \text { days) } \\
\text { Never/Very rarely (less than a day) } \\
\text { Very often ( } 3 \text { to } 4 \text { days) } \\
\text { Occasionally (I to } 2 \text { days) }\end{array}$ & $\begin{array}{c}8 \%(5) \\
47 \%(28) \\
8 \%(5) \\
37 \%(22)\end{array}$ & $\begin{array}{c}5 \%(6) \\
58 \%(67) \\
9 \%(10) \\
28 \%(33)\end{array}$ & $\begin{array}{l}6 \%(11) \\
54 \%(95) \\
9 \%(15) \\
31 \%(55)\end{array}$ & 0.495 \\
\hline
\end{tabular}

(Continued) 
Table 5 (Continued).

\begin{tabular}{|c|c|c|c|c|c|c|}
\hline Answer & $\begin{array}{c}\text { N. of } \\
\text { Responders }\end{array}$ & Response & $\begin{array}{l}\text { LEPRE } \\
(\mathrm{N}=64)\end{array}$ & $\begin{array}{l}\text { PRESTO } \\
(N=128)\end{array}$ & $\begin{array}{l}\text { Combined } \\
(\mathrm{N}=192)\end{array}$ & $\begin{array}{c}P \\
\text { value }\end{array}$ \\
\hline \multirow[t]{4}{*}{ I have been happy. } & \multirow[t]{4}{*}{176} & Frequently, all the time (5 to 7 days) & $7 \%(4)$ & $11 \%(13)$ & $10 \%(17)$ & 0.103 \\
\hline & & Never/Very rarely (less than a day) & $7 \%(4)$ & $17 \%(20)$ & $14 \%(24)$ & \\
\hline & & Very often ( 3 to 4 days) & $50 \%(30)$ & $35 \%(4 I)$ & $40 \%(7 I)$ & \\
\hline & & Occasionally ( 1 to 2 days) & $37 \%(22)$ & $36 \%(42)$ & $36 \%(64)$ & \\
\hline \multirow[t]{4}{*}{ I talked less than usual. } & \multirow[t]{4}{*}{176} & Frequently, all the time (5 to 7 days) & $3 \%(2)$ & $4 \%(5)$ & $4 \%(7)$ & 0.853 \\
\hline & & Never/Very rarely (less than a day) & $57 \%(34)$ & $58 \%(67)$ & $57 \%(101)$ & \\
\hline & & Very often ( 3 to 4 days) & $7 \%(4)$ & $9 \%(I I)$ & $9 \%(15)$ & \\
\hline & & Occasionally ( 1 to 2 days) & $33 \%(20)$ & $28 \%(33)$ & $30 \%(53)$ & \\
\hline \multirow[t]{4}{*}{ I felt alone. } & \multirow[t]{4}{*}{176} & Frequently, all the time (5 to 7 days) & $5 \%(3)$ & $4 \%(5)$ & $5 \%(8)$ & 0.95 \\
\hline & & Never/Very rarely (less than a day) & $48 \%(29)$ & $52 \%(60)$ & $51 \%(89)$ & \\
\hline & & Very often ( 3 to 4 days) & $17 \%(10)$ & $14 \%(16)$ & $15 \%(26)$ & \\
\hline & & Occasionally ( 1 to 2 days) & $30 \%(18)$ & $30 \%(35)$ & $30 \%(53)$ & \\
\hline \multirow{4}{*}{$\begin{array}{l}\text { Other people have been hostile } \\
\text { towards me. }\end{array}$} & \multirow[t]{4}{*}{176} & Frequently, all the time (5 to 7 days) & $2 \%(I)$ & $1 \%(I)$ & $1 \%(2)$ & 0.588 \\
\hline & & Never/Very rarely (less than a day) & $85 \%(5 I)$ & $78 \%(90)$ & $80 \%(|4|)$ & \\
\hline & & Very often ( 3 to 4 days) & $3 \%(2)$ & $5 \%(6)$ & $5 \%(8)$ & \\
\hline & & Occasionally ( $\mathrm{I}$ to 2 days) & $10 \%(6)$ & $16 \%(19)$ & $14 \%(25)$ & \\
\hline \multirow[t]{4}{*}{ I enjoyed life. } & \multirow[t]{4}{*}{176} & Frequently, all the time (5 to 7 days) & $12 \%(7)$ & $15 \%(17)$ & $14 \%(24)$ & 0.829 \\
\hline & & Never/Very rarely (less than a day) & $22 \%(13)$ & $26 \%(30)$ & $24 \%(43)$ & \\
\hline & & Very often ( 3 to 4 days) & $28 \%(17)$ & $26 \%(30)$ & $27 \%(47)$ & \\
\hline & & Occasionally ( 1 to 2 days) & $38 \%(23)$ & $34 \%(39)$ & $35 \%(62)$ & \\
\hline \multirow[t]{4}{*}{ I had crying fits. } & \multirow[t]{4}{*}{176} & Frequently, all the time (5 to 7 days) & $5 \%(3)$ & $2 \%(2)$ & $3 \%(5)$ & 0.471 \\
\hline & & Never/Very rarely (less than a day) & $62 \%(37)$ & $71 \%(82)$ & $68 \%(119)$ & \\
\hline & & Very often ( 3 to 4 days) & $10 \%(6)$ & $9 \%(I I)$ & $10 \%(17)$ & \\
\hline & & Occasionally ( 1 to 2 days) & $23 \%(14)$ & $18 \%(2 \mid)$ & $20 \%(35)$ & \\
\hline \multirow[t]{4}{*}{ I felt sad. } & \multirow[t]{4}{*}{176} & Frequently, all the time (5 to 7 days) & $5 \%(3)$ & $7 \%(8)$ & $6 \%(11)$ & 0.93 \\
\hline & & Never/Very rarely (less than a day) & $28 \%(I 7)$ & $28 \%(32)$ & $28 \%(49)$ & \\
\hline & & Very often ( 3 to 4 days) & $13 \%(8)$ & $16 \%(18)$ & $15 \%(26)$ & \\
\hline & & Occasionally ( 1 to 2 days) & $53 \%(32)$ & $50 \%(58)$ & $51 \%(90)$ & \\
\hline \multirow{4}{*}{$\begin{array}{l}\text { I have the impression that people } \\
\text { do not like me. }\end{array}$} & \multirow[t]{4}{*}{176} & Frequently, all the time (5 to 7 days) & $0 \%(0)$ & $3 \%(4)$ & $2 \%(4)$ & 0.029 \\
\hline & & Never/Very rarely (less than a day) & $93 \%(56)$ & $75 \%(87)$ & $81 \%(143)$ & \\
\hline & & Very often ( 3 to 4 days) & $2 \%(I)$ & $4 \%(5)$ & $3 \%(6)$ & \\
\hline & & Occasionally (I to 2 days) & $5 \%(3)$ & $17 \%(20)$ & $13 \%(23)$ & \\
\hline \multirow[t]{4}{*}{ I lack motivation. } & \multirow[t]{4}{*}{176} & Frequently, all the time (5 to 7 days) & $2 \%(I)$ & $5 \%(6)$ & $4 \%(7)$ & 0.095 \\
\hline & & Never/Very rarely (less than a day) & $57 \%(34)$ & $54 \%(63)$ & $55 \%(97)$ & \\
\hline & & Very often ( 3 to 4 days) & $22 \%(13)$ & $10 \%(12)$ & $14 \%(25)$ & \\
\hline & & Occasionally (I to 2 days) & $20 \%(12)$ & $30 \%(35)$ & $27 \%(47)$ & \\
\hline
\end{tabular}

Note: Significant $P$ values between LEPRE and PRESTO groups are expressed in bold characters.

patients with neuropsychiatric features were excluded. However, by excluding confounding factors such as different levels of disease activity as well as the presence of psychiatric features, the study selected a group of SLE patients as homogeneous as possible.
To conclude, restrictive measures for contrasting the COVID-19 pandemic did not unveil a greater psychological fragility of people living with SLE than the other people. By contrast, a coping strategy for managing a chronic autoimmune disease, including the role of the family and the 


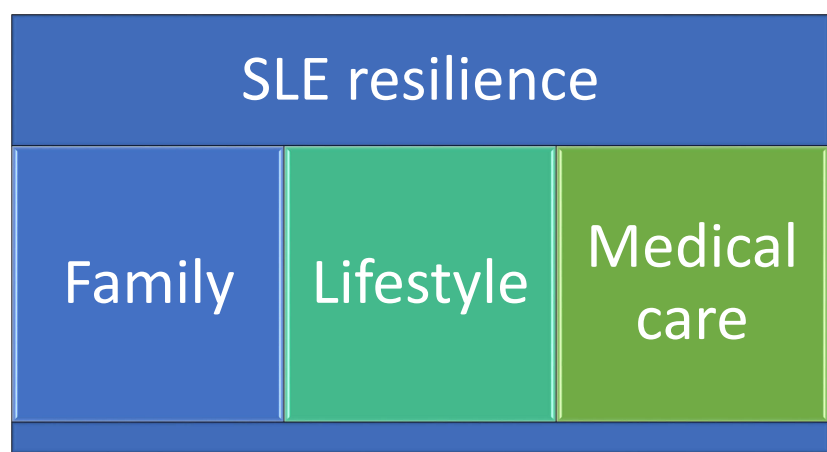

Figure I The three pillars of lupus patients' resilience.

lifestyle, contributes to resilience of SLE in difficult scenarios such as those presented by the pandemic.

\section{Data Sharing Statement}

All data relevant to the study are included in the article or uploaded as Supplemental Materials. The online questionnaire is available at https://www.prestoinsieme.com. We used deidentified participant data.

\section{Ethical Approval Information}

The Italian "Istituto Superiore di Sanità" (ISS) Ethics Committee has took notice of the study, since it was only a survey collecting anonymized data. It followed the European and the Italian privacy policy. People participating to the survey digitally gave consent before starting the questionnaire.

\section{Acknowledgments}

Local patients' associations for rheumatic diseases and stakeholders other than physicians (psychologist, health professional, nutritionist, public health scientist) were involved in identifying this topic as an urgent need for the best management of patients suffering from systemic lupus erythematosus as a model of the autoimmune diseases, and to deeper understand the multidimensional features of the disease, in difficult context such as the outbreak and the restrictive measures. This information has been thought of relevance also for improving the quality of care of patients in general, and not only in the present outbreak.

\section{Funding}

There are no funders to report for this submission.

\section{Disclosure}

No authors have any financial support or other benefits from commercial sources for the work reported on in the manuscript, or any other financial interests which could create a potential conflict of interest or the appearance of a conflict of interest concerning the work. The authors reported no conflicts of interest for this work.

\section{References}

1. Huang C, Wang Y, Li X, et al. Clinical features of patients infected with 2019 novel coronavirus in Wuhan, China. Lancet. 2020;395:497-506. doi:10.1016/S0140-6736(20)30183-5

2. Gianfrancesco M, Hyrich KL, Al-Adely S, et al. Characteristics associated with hospitalisation for COVID-19 in people with rheumatic disease: data from the COVID-19 Global Rheumatology Alliance physician-reported registry. Ann Rheum Dis. 2020;79:859866. doi:10.1136/annrheumdis-2020-217871

3. Booth S, Price E. Fluctuation, invisibility, fatigue - the barriers to maintaining employment with systemic lupus erythematosus: results of an online survey. Lupus. 2018;27:2284-2291. doi:10.1177/ 0961203318808593

4. Abu-Shakra M. Quality of life, coping and depression in systemic lupus erythematosus. IMAJ. 2016;18:144-145.

5. Isung J, Williams K, Isomura $\mathrm{K}$, et al. Association of primary humoral immunodeficiencies with psychiatric disorders and suicidal behavior and the role of autoimmune diseases. JAMA Psychiatry. 2020;77(11):1-9. doi:10.1001/jamapsychiatry.2020.1260

6. Brooks SK, Webster RK, Smith LE, et al. The psychological impact of quarantine and how to reduce it: rapid review of the evidence. Lancet. 2020;395:912-992. doi:10.1016/S0140-6736(20)30460-8

7. Aringer M, Costenbader K, Daikh D, et al. 2019 European League Against Rheumatism/American College of Rheumatology classification criteria for systemic lupus erythematosus. Arthritis Rheumatol. 2019;71(9):1400-1412. doi:10.1002/art.40930

8. Golder V, Tsang-A-Sjoe MWP. Treatment targets in SLE: remission and low disease activity state. Rheumatology (Oxford). 2020;59 (Supp15):v19-v28. doi:10.1093/rheumatology/keaa420.

9. Weiss DS, Marmar CR. The impact of event scale - revised. In: Wilson JP, Keane TM, editors. Assessing Psychological Trauma and PTSD. New York: Guilford Press; 1997:399-411.

10. Goldberg DP, Gater R, Sartorius N, et al. The validity of two versions of the GHQ in the WHO study of mental illness in general health care. Psychol Med. 1997;27(1):191-197. doi:10.1017/S003329179 6004242

11. Radloff LS. The CES-D scale: a self-report depression scale for research in the general population. Appl Psychol Meas. 1977;1:385401. doi:10.1177/014662167700100306

12. Wang $\mathrm{C}$, Pan $\mathrm{R}$, Wan $\mathrm{X}$, et al. Immediate psychological responses and associated factors during the initial stage of the 2019 Coronavirus Disease (COVID-19) Epidemic among the General Population in China. Int J Environ Res Public Health. 2020;17:1729. doi:10.3390/ ijerph17051729

13. Giorgi G, Perez JML, D'Antonio AC, et al. The general health questionnaire (GHQ-12) in a sample of Italian workers: mental health at individual and organizational level. World J Med Sci. 2014;11:47-56.

14. Zhang Z, Kim HJ, Lonjon G, Zhu Y. Balance diagnostics after propensity score matching. Ann Transl Med. 2019;7:16. doi:10.21 037/atm.2018.12.10

15. George M, Venkatachalam S, Banerjee S, et al. Concerns, healthcare use, and treatment interruptions in patients with common autoimmune rheumatic diseases during the COVID-19 pandemic. $J$ Rheumatol. 2020. doi:10.3899/jrheum.201017.

16. Koppert TY, Jacobs JWG, Geenen R. The psychological impact of the COVID-19 pandemic on Dutch people with and without an inflammatory rheumatic disease. Rheumatology. 2020;keaa842. doi:10.1093/rheumatology/keaa842. 
17. Wańkowicz P, Szylińska A, Rotter I. Evaluation of mental health factors among people with systemic lupus erythematosus during the SARS-CoV-2 pandemic. J Clin Med. 2020;9(9):2872. doi:10.3390/ jcm 9092872

18. Tee CA, Salido EO, Reyes PWC, Ho RC, Tee ML. Psychological state and associated factors during the 2019 Coronavirus Disease (COVID-19) pandemic among filipinos with rheumatoid arthritis or systemic lupus erythematosus. Open Access Rheumatol. 2020;12:215-222. doi:10.2147/OARRR.S269889
19. Santos-Ruiz A, Montero-López E, Ortego-Centeno N, PeraltaRamírez MI. Effect of COVID-19 confinement on the mental status of patients with systemic lupus erythematosus. Med Clin (Engl Ed). 2021;156(8):379-385. doi:10.1016/j.medcle.2020.12.009.

20. Faria DA, Revoredo LS, Vilar MJ, Eulália Maria Chaves M. Resilience and treatment adhesion in patients with systemic lupus erythematosus. Open Rheumatol J. 2014;8:1-8. doi:10.2174/ 1874312920140127001

\section{Publish your work in this journal}

The Journal of Multidisciplinary Healthcare is an international, peerreviewed open-access journal that aims to represent and publish research in healthcare areas delivered by practitioners of different disciplines. This includes studies and reviews conducted by multidisciplinary teams as well as research which evaluates the results or conduct of such teams or healthcare processes in general. The journal covers a very wide range of areas and welcomes submissions from practitioners at all levels, from all over the world. The manuscript management system is completely online and includes a very quick and fair peer-review system. Visit http://www.dovepress.com/testimonials. php to read real quotes from published authors. 